\title{
Fabric Defect Detection based on GLCM
}

\author{
Zhang Xiaowei ${ }^{1, a^{*}}$, Fan Xiujuan ${ }^{2, b}$ \\ ${ }^{1}$ Chaoyang district, Beijing, China Beijing Institute of Fashion Techology \\ ${ }^{2}$ Chaoyang district, Beijing, China Beijing Institute of Fashion Techology \\ a602256515@qq.com, b gxyfanxj@bift.edu.cn
}

Keywords: GLCM; Fabric defect detection; texture features

Abstract:In general, an image of woven fabric sample can be regarded as a typical textured image. The detection of local fabric defects is one of the most captivating problems in computer vision and has received much attention over the years. In the textile industry, careful inspections for woven fabrics have to be carried out because fabric defects may reduce the profit of a company by $45 \%$ or $65 \%$. Real time automated fabric defect detection plays a crucial role in the textile manufacturing industry to ensure that the industry meets its high quality standards. Indeed. The production of good quality products is a key issue for increasing profitability and customer satisfaction and thus improving the industry's competitive edge in the global market. If defects in the fabrics are not discovered prior to the garment manufacturing process, significant financial losses can incur.

\section{Introduction}

Typically web textile fabric is 1-3 m wide and is driven with speed ranging from 20 to 200 $\mathrm{m} / \mathrm{min}$. At present, the quality inspection process is manually preformed by experts. However they cannot detect more than $60 \%$ of the overall defects for the fabric if it is moving faster than 30 $\mathrm{m} / \mathrm{min}$. To increase the quality and homogeneity of fabrics, an automated visual inspection system is needed for better productivity. One way to reduce the total manufacturing cost and to provide a more reliable, objective , and consistent quality control process is to use an automated visual inspection system to detect possible defects in textile fabrics. However, automated visual inspection becomes a significant challenge due to some specific features pertaining to textile fabrics, for example:

(a) Large variety of fabric surfaces has to be examined.

(b) Defects may take different forms that are usually difficult to classify.

(c) New classes of defects arising from possible changes or aging of

machineries in the production process.

This paper proposes a new GLCM based technique to address the problem. A sample of different kind of texture defects is shown in Fig. 1. The paper is organized as follows: Section 2introduction the GLCM while Sections 3 describe defect detection system implementation of GLCM approach. Section 4 presents results and discussion.

Finally, Section 5 draws the conclusion of the research.
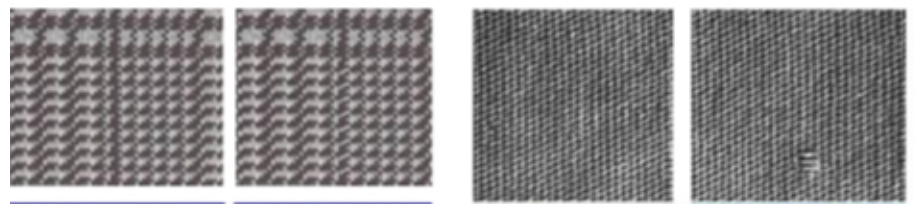

Fig. 1. Non-defective and defective fabric images 


\section{GLCM}

The co-occurrence probabilities provide a second order method for generating texture features. A brief presentation of the GLCM method follows but a more complete explanation is provided by Haralick. The matrix contains the conditional joint probabilities of all pair wise combinations of gray levels given two parameters: interpixel distance (d) and interpixel orientation $(\theta)$. Following Barber, the probability measure can be defined as:

$$
p_{r}(x)=\left\{C_{i j} \mid(d, \theta)\right\}
$$

where $\mathrm{Cij}$ (the GLCM) is defined as:

$$
C_{i j}=\frac{P_{i j}}{\sum_{i, j=1}^{G} P_{i j}}
$$

Pij represents the number of occurrences of gray levels gi and gj and $\mathrm{G}$ is the total number of gray levels. The sum in the denominator represents the total number of gray level pairs within a window given a particular $(\mathrm{d}, \theta)$. A different GLCM is required for each (d, $\theta)$. Pixel separation distances (d) are usually less than 10. Typically, only four orientations are used for the simplicity of computation: 0, 45, 90 and 135 degrees. The orientation 180 degrees is redundant to 0 degrees, the orientation 225 degrees is redundant to 45 degrees, etc.

The average of the four orientations may be used if invariance to spatial rotations is desired. Different statistical information can be determined from each GLCM; however, statistics that are gray level shift invariant are important so that the classification is not a function of tone. Eight such shift invariant statistics are presented in Table 1.

Table 1 GLCM texture statistics defined.

\begin{tabular}{|l|c|}
\hline Maximum probability (MAX) & $\max \left\{c_{i j} \forall(i, j)\right\}$ \\
\hline Energy (ENG) & $\sum_{i, j=1}^{G} c_{i, j}^{2}$ \\
\hline Entropy (ENT) & $-\sum_{i, j=1}^{G} c_{i j} \log c_{i j}$ \\
\hline Dissimilarity (DIS) & $\sum_{i, j=1}^{G} c_{i j|i-j|}$ \\
\hline Contrast (CON) & $\sum_{i, j=1}^{G} C_{i j(i-j)^{2}}$ \\
\hline Homogeneity (HOM) & $\sum_{i, j=1}^{G} \frac{1}{1+|i-j|} C_{i j}$ \\
\hline Inverse Difference Moment (IDM) & $\sum_{i, j=1}^{G} \frac{1}{1+(i-j)^{2} / G^{2}} C_{i j}$ \\
\hline Correlation (COR) & $\sum_{i, j=1}^{G} \frac{\left(i-u_{i}\right)\left(j-u_{j}\right)}{\sigma \sigma_{j}} C_{i j}$ \\
\hline
\end{tabular}

\section{Defect detection system implementation of GLCM approach}

Fig. 2 describes the GLCM based defect detection approach proposed in this paper. In this study, GLCM is computed based on two parameters, which are the distance between the pixel pair $\mathrm{d}$ and their angular relation $\theta$. Generally earlier studies quantized $\theta$ in four directions $\left(0^{\circ}, 45^{\circ}, 90^{\circ}\right.$ and $135^{\circ}$ ). Typically, the GLCM is calculated in a small window, which scans the whole image. 
Before calculating GLCM, all the defective and non-defective images should be converted to gray scale. The test images acquired from camera are of 512 × 512 resolutions. The resulting GLCM are computed over several displacements, in which the distances may vary from 1 pixel to full dynamic range and the angle is $0^{\circ}$. Before texture calculation, the GLCM is normalized and then GLCM represents probabilities instead of counts. Normalization involves dividing by the total number of counted pixel pairs.

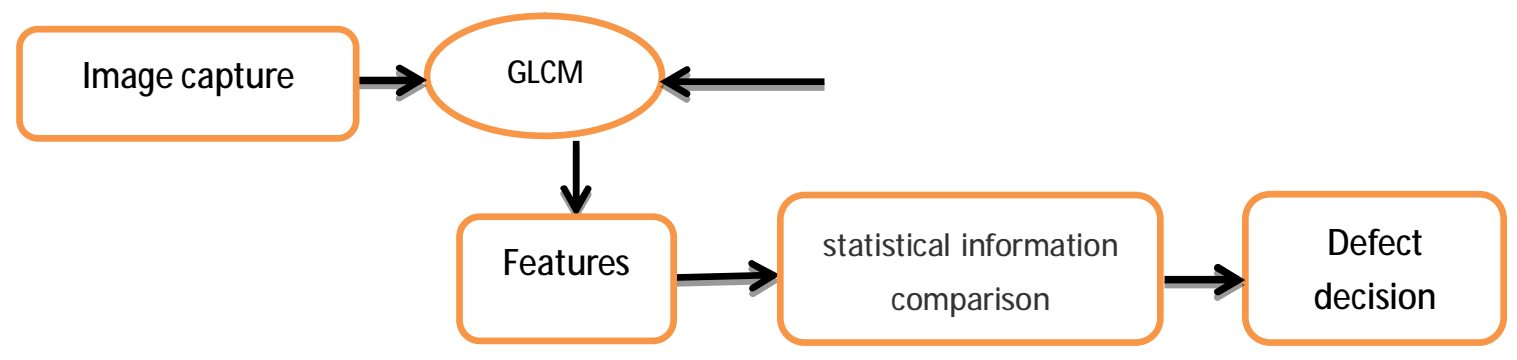

Fig. 2 Defect detection using GLCM.

Only horizontal offset considered makes the algorithm fast. In this algorithm, four GLCM features are considered: ENG, HOM, CON and COR as these four features gave the best results for smaller window size in defect detection scheme. For each d value, four elemental feature matrices (EFM) are derived. This algorithm is faster than earlier one as it considers only horizontal offsets with small window size of 10x10 Now, calculate the various statistical information of non-defective template image using four features by applying different values of $\mathrm{d}$. Same is done for acquired test images and the statistical information are compared. If they are same, there is no defect otherwise the sample is defected.

\section{Results and discussion}

The performance of the proposed defect detection scheme is evaluated by using an offline test database containing 60 fabric images acquired from different sources. In the database, 30 images are defect-free and the other 30 are of defects commonly appearing in textile fabrics with a variety of shapes as shown in Fig. 1. These defects are caused by different reasons, including missing yards, broken yards, and changes in tension during production in a textile loom. The fabrics in the database are mainly plain, twill, denim weave fabrics, although other types of fabrics are also included. In this test, the fabric images are 512 x 512 pixels in size with 8 bit gray level. The main three parameters considered are $\mathrm{d}=1, \theta=0^{\circ}$ and different values of windows were taken. We considered $10 \times 10,20 \times 20,50 \times 50$ and $60 \times 60$. Window size is to be determined based on two opposing factors, number of computations and precision of localization of the defect. The test results of some defects are shown in Fig.3 which commonly appear in textile and garment manufacturing industries. 

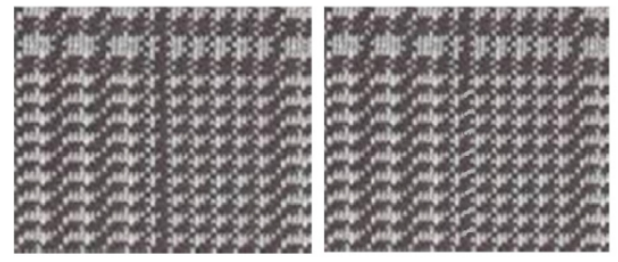

Contrast difference $=-\mathbf{- 0 . 0 5 2 5}-\mathbf{- 0 . 0 5 3 5}$

Energy difference $=0.0002 \quad 0.0012 \quad 0.0003$

Homogeneity difference $=\mathbf{0 . 0 0 1 3}$

Correlation difference $=\mathbf{0 . 0 0 2 4}$
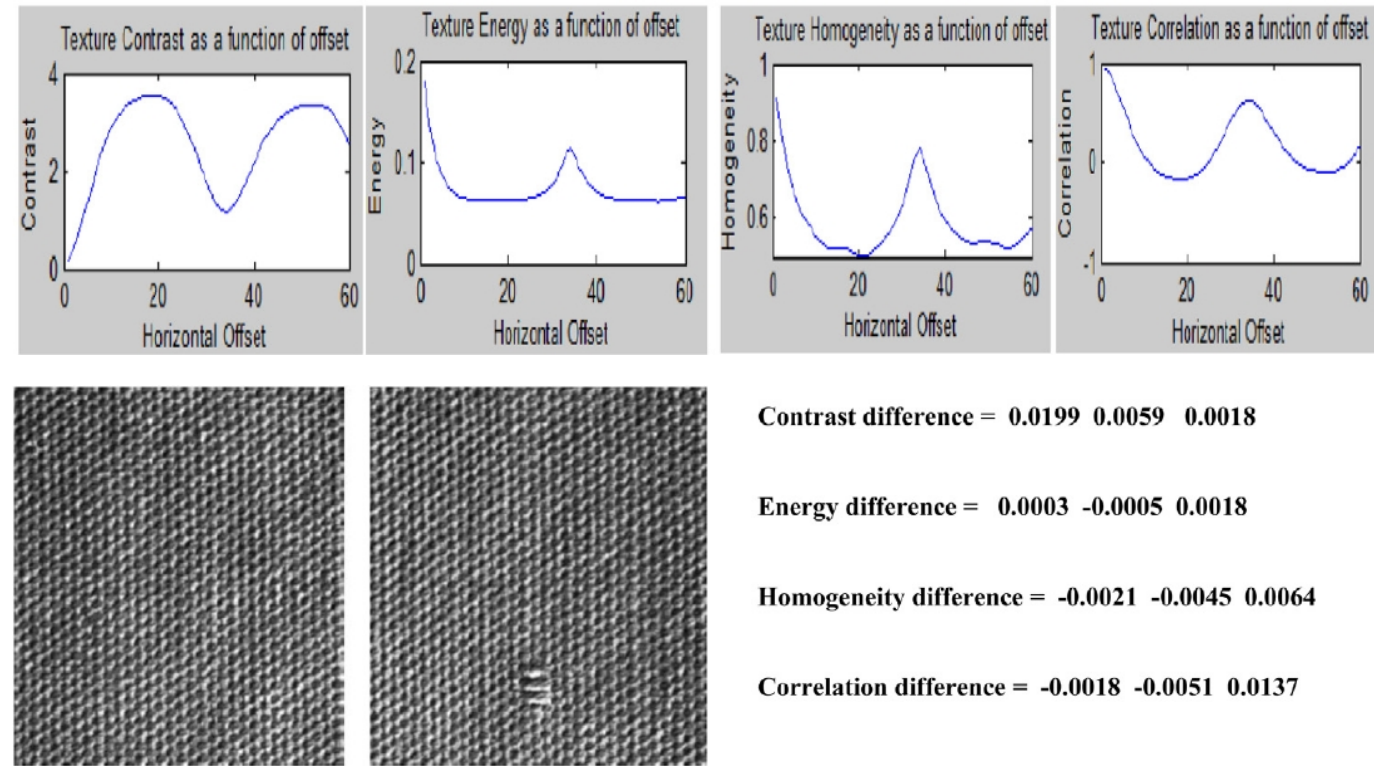

Contrast difference $=0.01990 .00590 .0018$

Energy difference $=0.0003-0.00050 .0018$

Homogeneity difference $=-\mathbf{- 0 . 0 0 2 1}-\mathbf{- 0 . 0 0 4 5} 0.0064$

Correlation difference $=-\mathbf{0 . 0 0 1 8}-\mathbf{- 0 . 0 0 5 1} 0.0137$
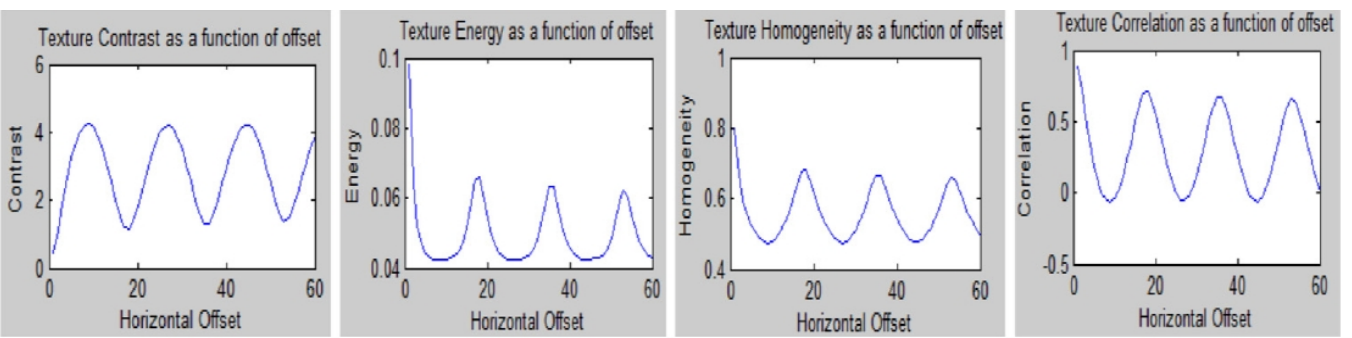

Fig. 3. Results of GLCM method: non-defective and defective fabric images with the four features differences and their graph.

In the Fig. 3, the images on the left are non-defective template image and the images on the right column are defective images. It can be clearly seen that the proposed scheme can detect both global defects and local defects. Statistical information of the four GLCM texture features are obtained for both the images and plotted against $C$ with value 60 . The offset location at which the peaks occur gives the periodicity of that property in the image. If there is difference in the peaks of various features along horizontal offset, then there is a defect in the fabric. Difference in peaks of various features is shown below the images in Fig. 3. Table 2 shows the efficiency of detection. TD is true detection, FD is false detection, MD is missed detection and TI is sum of TD and FD.

Table 2 Performance evaluation of GLCM approach.

\begin{tabular}{|l|l|l|l|c|}
\hline & TI & TD & FD & MD \\
\hline numbers & 60 & 59 & 1 & 0 \\
\hline$\%$ & 100 & 98.33 & 1.67 & 0 \\
\hline
\end{tabular}

\section{Conclusion}

In general, defect detection approaches can be classified into two categories: supervised and unsupervised detection approach. Practically a conventional supervised approach based on the 
knowledge of some particular defect types may not be suitable. On the other hand, the design of an unsupervised approach is rather complicated. The performances of the schemes developed have been extensively evaluated by using an offline test database, which contains a variety of fabric images, with and without defects chosen from various sources. The output results obtained from experiments clearly show that most of the defects can be successfully detected. These excellent results clearly indicate that the proposed schemes are an effective and robust means for detecting fabric defects, but GLCM scheme has also a disadvantage that it can work only in invariant environmental condition.

\section{References}

[1] K. Srinivasan, P.H. Dastoor, P. Radhakrishnaiah, S. Jayaraman, FDAS: A knowledge-based framework for analysis of defects in woven textile structures, J. Textile Inst. 83 (3 (pt. 1)) (1992) 431-448.

[2] C. Cho, B. hung, M. Park, Development of Real-Time Vision-Based Fabric Inspection System, IEEE Trans. Ind. Electron. 52 (4 (August)) (2005) 1073-1079.

[3] Y.A. Karayiannis, R. Stojanovic, P. Mitropoulos, C. Koulamas, T. Stouraitis, S. Koubias, G. Papadopoulos, Defect detection and classification on web textile fabric using multiresolution decomposition and neural networks, in: Proceedings of 6th IEEE International Conference on Electronics, Circuits and Systems, 5-8 September, 1999, pp. 765-768.]

[4] R.M. Haralick, Statistical and structural approaches to texture, in: Proceedings of the IEEE, vol. 67, 1979, pp. 786-804.

[5] R.M. Harlick, K. Shanmugam, I. Dinstein, IEEE trans, Sys. Man Cybern. 3 (1973) 610-621.

[6] D.G. Barber, E.F. LeDrew, SAR sea ice discrimination using texture statistics: a multivariate approach, Photogrammetric Engineering and Remote Sensing 57

(4 (April)) (1991) 385-395.

[7] J. Daugman, Uncertainty relation for resolution in space, spatial frequency, and orientation optimized by two-dimensional visual cortical filters, J. Opt. Soc. Am. 2 (7) (1985) 1160-1169.

[8] B.S. Manjunath, W.Y. Ma, Texture features for browsing and re-trieval of image data, IEEE Trans. Pattern Anal. Machine Intell. 18 (8 (August)) (1996) 837-842. 